\title{
Letter Regarding: Selenium and Preeclampsia: A Systemic Review and Meta-Analysis
}

\author{
Hiten D. Mistry ${ }^{1}$ - Fiona Broughton Pipkin ${ }^{1}$ Lesia O. Kurlak ${ }^{1}$
}

Received: 8 December 2015 / Accepted: 25 January 2016 / Published online: 29 January 2016

(C) Springer Science+Business Media New York 2016

Sir,

We are surprised to see several serious errors have crept into systematic review and meta-analysis on selenium and preeclampsia (PE) by Xu et al., (October 2015) [1], detailed below.

First, we believe they have misinterpreted our data published in Hypertension [2]. The authors state that 'Se in the umbilical cord was significantly lower in the PE group than in the control while not in serum in two studies [25, 26]' where reference 26 is our manuscript. But our data show that the umbilical venous serum selenium concentrations were lower in the PE group: 'Selenium concentrations were significantly reduced in umbilical venous samples in preeclampsia by comparison with samples from babies of normotensive mothers $(P<0.0001)^{\prime}$. Secondly, the numbers quoted in the manuscript Table 1 and Figure 2 are incorrect and should be (mean \pm SD) normotensive 42.1 \pm 11.8 ; PE $29.0 \pm 9.9 \mu \mathrm{g} / \mathrm{L}$; these have been written the wrong way around and thus significantly affects the results of the authors analyses, particularly in relation to the data presented in Figure 2.

In addition to the above, in both our manuscripts [2;3], we have clearly stated the PE criteria, which are detailed in the methods. Finally, the mean age (year) for the women used in our study is published in Table 1 of the manuscript [3].

We would be grateful of some acknowledgement to the original manuscript that the error will affect the systematic review and meta-analysis results. On finding these errors, we are concerned that similar errors may have occurred to other manuscripts referenced in this systematic review/metaanalysis.

\section{References}

1. Xu M, Guo D, Gu H, Zhang L, Lv S (2015) Selenium and preeclampsia: a systematic review and meta-analysis. Biol Trace Elem Res. doi:10.1007/s12011-015-0545-7

2. Mistry HD, Wilson V, Ramsay MM, Symonds ME, Broughton Pipkin F (2008) Reduced selenium concentrations and glutathione peroxidase activity in pre-eclamptic pregnancies. Hypertension 52 : 881-888

3. Mistry HD, Gill CA, Kurlak LO, et al. (2015) Association between maternal micronutrient status, oxidative stress, and common genetic variants in antioxidant enzymes at 15 weeks gestation in nulliparous women who subsequently develop preeclampsia. Free Radic Biol Med 78:147-155

Hiten D. Mistry

hiten.mistry@nottingham.ac.uk

1 Division of Child Health, Obstetrics \& Gynaecology, School of Medicine, University of Nottingham, 1st Floor, Maternity Unit, City Hospital, Hucknall Road, Nottingham NG5 1PB, UK 